\title{
РЕЗУЛЬТАТЫ ВНЕДРЕНИЯ НОВЫХ МЕТОДОВ ТРАНСУРЕТРАЛЬНОЙ БИПОЛЯРНОЙ ЭНДОХИРУРГИИ В ЛЕЧЕНИИ ДОБРОКАЧЕСТВЕННОЙ ГИПЕРПЛАЗИИ ПРОСТАТЫ
}

\section{RESULTS OF INTRODUCTION OF NEW METHODS OF TRANSURETHRAL BIPOLAR ENDOSURGERY IN THE TREATMENT OF BENIGN PROSTATIC HYPERPLASIA}

P. Kononov A. Kurnakov

Summary. New methods of transurethral bipolar surgery for the treatment of patients with benign prostatic hyperplasia (BPH) have been introduced in the urology Department of the Russian Railways Road hospital in Saint Petersburg since 2018. With prostate volumes up to $80 \mathrm{~cm} 3$, bipolar transurethral resection (BTUR) is performed, and over $80 \mathrm{~cm} 3$ - bipolar transurethral enucleation of the prostate (TUEB). 114 patients were selected for the study, divided into groups according to the methods of surgery. The main groups consisted of 29 patients operated by the BTUR method, and 27 by the TUEB method. The control groups included 27 patients operated by the method of monopolar TUR (MTUR), and 31 by the method of open transvesical adenomectomy (OAE), performed in similar clinical situations before the introduction of new surgical methods in the Department. Comparison with the control showed a significant reduction in blood loss during surgery, the duration of postoperative catheterization of the bladder, the frequency of hemorrhagic and infectious complications, as well as the number of postoperative bed days for bipolar methods. A decrease in the frequency of delayed obstructive complications was also observed for BTUR in comparison with MTUR. The postoperative dynamics of urological indicators for all groups was positive, but the examination of patients 1 month after BTUR and TUEB showed a faster recovery of impaired urination parameters. This study allowed us to evaluate the implementation and recommend it to other urological hospitals in order to improve the quality of surgical care for patients with BPH and save costs by reducing the postoperative bed day.

Keywords: benign hyperplasia of the prostate, bipolar transurethral resection and enucleation of the prostate.

\author{
Кононов Павел Алексеевич \\ Врач-уролог, НУз Дорожная клиническая больница \\ ОАО «РЖД», г. Санкт-Петербург \\ pavkonon@gmail.com \\ Курнаков Антон Михайлович \\ Врач уролог, НУЗ Дорожная клиническая больница \\ ОАО «РЖД», г. Санкт-Петербург
}

Аннотация. В урологическом отделении Дорожной клинической больницы ОАО «РЖД» г. Санкт-Петербурга с 2018 года внедрены новые методы трансуретральных биполярных операций для лечения пациентов с доброкачественной гиперплазией простаты (ДГП). При объемах простаты до 80 см3 выполняется биполярная трансуретральная резекция (БТУР), свыше 80 см3 — биполярная трансуретральная энуклеация простаты (ТУЭБ). Для исследования отобрано 114 пациентов, разделенных на группы по методам операции. Основные группы составили 29 пациентов, оперированных методом БТУР, и 27 - методом ТУЭБ. В контрольные группы включены 27 пациентов, оперированных методом монополярной ТУР (МТУР), и 31 - методом открытой чреспузырной аденомэктомии (ОАЭ), выполняемых при подобных клинических ситуациях до внедрения в отделении новых хирургических методов. Сравнение с контролем показало для биполярных методов значимое сокращение объема кровопотери во время операций, сроков послеоперационной катетеризации мочевого пузыря, частоты геморрагических и инфекционных осложнений, а также числа послеоперационных койко-дней. Для БТУР в сравнении с МТУР также отмечено снижение частоты отсроченных обструктивных осложнений. Послеоперационная динамика урологических показателей для всех групп была положительной, но обследование пациентов через 1 месяц после БтУР и ТУэБ показало более быстрое восстановление нарушенных параметров мочеиспускания. Настоящее исследование позволило оценить проведенное внедрение и рекомендовать его другим урологическим стационарам в целях повышения качества хирургической помощи пациентам с ДГП и экономии средств за счет снижения послеоперационного койко-дня.

Ключевые слова: доброкачественная гиперплазия простаты, биполярная трансуретральная резекция и энуклеация простаты.

В соответствии с клиническими рекомендациями в активных методах лечения нуждаются лица с выраженной клинической симптоматикой ДГП, соответствующей 8 баллам и выше по международной шкале оценки простатических симптомов - IPSS $[8,10]$. В настоящее время в числе активных методов лечения наиболее часто ис- 
Таблица 1. Предоперационные данные пациентов (медианные показатели Ме $\pm \mathrm{m}$ в соответствующих единицах измерения; осложнения и сопутствующие заболевания —\% в группе $\mathrm{P} \pm \mathrm{m}$ )

\begin{tabular}{|c|c|c|c|c|c|c|}
\hline $\begin{array}{l}\text { Метод } \\
\text { операции }\end{array}$ & $\begin{array}{l}\text { БТУР } \\
\mathrm{N}=29\end{array}$ & $\begin{array}{l}\text { MTУP } \\
N=27\end{array}$ & P* & $\begin{array}{l}\text { ТУЭБ } \\
\mathrm{N}=27\end{array}$ & $\begin{array}{l}\mathrm{OA} \ni \\
\mathrm{N}=31\end{array}$ & $\mathbf{P}^{*}$ \\
\hline Возраст, лет & $61,2 \pm 3,37$ & $60,4 \pm 3,41$ & 0,868 & $66,5 \pm 3,58$ & $65,1 \pm 3,42$ & 0,778 \\
\hline уЗИ объем ПЖ, см³ & $65,5 \pm 3,49$ & $63,6 \pm 3,50$ & 0,702 & $92,2 \pm 4,21$ & $91,1 \pm 4,05$ & 0,851 \\
\hline Уровень ПСА, нг/мл & $3,6 \pm 0,82$ & $3,2 \pm 0,78$ & 0,725 & $3,8 \pm 0,86$ & $3,9 \pm 0,84$ & 0,934 \\
\hline Объем остаточной мочи, PVR, мл & $69,2 \pm 3,50$ & $63,1 \pm 3,51$ & 0,650 & $100,8 \pm 4,39$ & $104,3 \pm 4,26$ & 0,644 \\
\hline $\begin{array}{l}\text { Скорость потока мочи, Qmax, мл/ } \\
\text { сек }\end{array}$ & $10,1 \pm 1,37$ & $9,6 \pm 1,36$ & 0,797 & $8,2 \pm 1,26$ & $7,7 \pm 1,18$ & 0,772 \\
\hline Балл по шкале IPSS & $25,1 \pm 2,16$ & $23,7 \pm 2,14$ & 0,647 & $29,3 \pm 2,37$ & $28,6 \pm 2,27$ & 0,832 \\
\hline Балл по шкале QOL & $4,1 \pm 0,87$ & $4,0 \pm 0,88$ & 0,936 & $5,4 \pm 1,02$ & $5,1 \pm 0,96$ & 0,831 \\
\hline \multicolumn{7}{|l|}{ Осложнения ДГП (\% в группе): } \\
\hline Камни МП & $13,8 \pm 6,52$ & $11,1 \pm 6,16$ & 0,765 & $40,7 \pm 9,64$ & $51,6 \pm 8,98$ & 0,410 \\
\hline Эпицистостома & $3,5 \pm 3,45$ & $0,0 \pm 0,00$ & 0,322 & $14,8 \pm 6,97$ & $19,4 \pm 7,10$ & 0,647 \\
\hline Острая задержка мочи & $3,5 \pm 3,45$ & $3,7 \pm 3,70$ & 0,960 & $33,3 \pm 9,25$ & $25,8 \pm 7,86$ & 0,538 \\
\hline Дивертикул МП & $6,9 \pm 4,79$ & $11,1 \pm 6,16$ & 0,591 & $18,5 \pm 7,62$ & $9,7 \pm 5,31$ & 0,346 \\
\hline Двусторонний гидронефроз & $3,5 \pm 3,45$ & $0,0 \pm 0,00$ & 0,322 & $7,4 \pm 5,14$ & $3,2 \pm 3,17$ & 0,493 \\
\hline \multicolumn{7}{|c|}{ Сопутствующие заболевания (\% в группе): } \\
\hline Гипертоническая болезнь & $51,7 \pm 9,44$ & $44,4 \pm 9,75$ & 0,591 & $66,7 \pm 9,25$ & $64,5 \pm 8,59$ & 0,866 \\
\hline Постинфарктный кардиосклероз & $10,3 \pm 5,76$ & $7,4 \pm 5,14$ & 0,705 & $14,8 \pm 6,97$ & $6,5 \pm 4,41$ & 0,317 \\
\hline Цереброваскулярные болезни & $3,5 \pm 3,45$ & $7,4 \pm 5,14$ & 0,525 & $11,1 \pm 6,16$ & $6,5 \pm 4,41$ & 0,544 \\
\hline Сахарный диабет & $10,3 \pm 5,76$ & $3,7 \pm 3,70$ & 0,336 & $18,5 \pm 7,62$ & $16,1 \pm 6,61$ & 0,811 \\
\hline Прием антикоагулянтов & $13,8 \pm 6,52$ & $7,4 \pm 5,14$ & 0,445 & $37,0 \pm 9,47$ & $22,6 \pm 7,51$ & 0,235 \\
\hline
\end{tabular}

* - принятое значение достоверности различий $\mathrm{p} \leq 0,05$, в таблице по всем измеряемым параметрам значение $\mathrm{p}>0,05$, что свидетельствует об отсутствии различий и статистической сопоставимости групп пациентов.

пользуют малоинвазивные хирургические методы, пришедшие на смену открытых травматичных вмешательств на простате [6,20]. В начале века в арсенале урологов появилась биполярная (плазменная) техника выполнения трансуретральных операций при ДГП, в частности биполярная трансуретральная резекция (БТУР), заменившая монополярную технику выполнения трансуретральной резекции, сопряженную с развитием ТУР-синдрома, массивными кровотечениями и электроожогами $[3,18,19]$. БТУР показана при объемах гиперплазии простаты до $80 \mathrm{~cm}^{3}$, и особенно при наличии осложнений ДГП, тяжелой интеркуррентной патологии и высокого геморрагического риска $[1,2,12]$. При более крупных размерах железы рекомендуется выполнение трансуретральной биполярной энуклеации простаты (ТУЭБ), которая по результатам множества сравнительных исследований, не менее эффективна, чем стандартная открытая чреспузырная аденомэктомия (ОАЭ), но при этом отличается минимальной инвазивностью, низким процентом геморрагических осложнений и более короткими сроками восстановления самостоятельного мочеиспускания, общего состояния и качества жизни пациентов после операции [5,11,13,14,16,]. Важным преимуществом ТУЭБ также считают малое количество противопоказаний и отсутствие ограничений по объему гиперплазирован- ной ткани, что позволяет выполнять данные операции при крупных и даже гигантских (свыше $150 \mathrm{~cm}^{3}$ ) размерах ДГП, а также при необходимости симультанных вмешательств $[7,9,17]$.

Целью проведенного исследования было оценить клиническую эффективность биполярных методов трансуретральных операций, выполняемых при лечении ДГП вместо традиционных МТУР и ОАЭ.

\section{Материалы и метолы}

Выбор биполярного метода трансуретральной резекции либо энуклеации простаты определялся дифференцированно в зависимости от объема железы, а также от строения и конфигурации гиперплазированной ткани, наличия осложнений ДГП, анестезиологического и геморрагического риска. Для исследования было отобрано 114 пациентов, разделенных на группы по методам оперативного вмешательства. Средний возраст пациентов составил $63,4 \pm 3,40$ (от 44 до 83 лет). Группу БТУР составили 29 пациентов с объемом простаты до $80 \mathrm{~cm}^{3}$, равным в среднем $65,5 \pm 3,49 \mathrm{~cm}^{3}$ (от 32 до $80 \mathrm{~cm}^{3}$ ), группу ТУЭБ - 27 пациентов с объемом железы свыше $80 \mathrm{~cm}^{3}$, в среднем 92,2 $44,21\left(92 / 128\right.$ см$\left.^{3}\right)$. Контрольные группы 


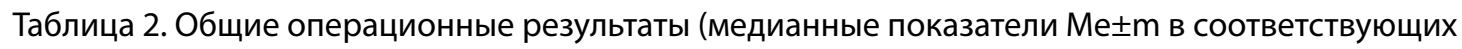
единицах измерения)

\begin{tabular}{|l|l|l|l|l|l|l|}
\hline $\begin{array}{l}\text { Метод } \\
\text { операции }\end{array}$ & $\begin{array}{l}\text { БТУР } \\
\mathbf{N = 2 9}\end{array}$ & $\begin{array}{l}\text { МТУР } \\
\mathbf{N = 2 7}\end{array}$ & $\mathbf{P}$ & $\begin{array}{l}\text { ТУЭБ } \\
\mathbf{N = 2 7}\end{array}$ & $\begin{array}{l}\text { ОАЭ } \\
\mathbf{N}=\mathbf{3 1}\end{array}$ & $\mathbf{P}$ \\
\hline Время операции & $40,1 \pm 2,73$ & $38,1 \pm 2,71$ & 0,605 & $76,2 \pm 3,83$ & $48,2 \pm 2,94$ & $<0,001^{*}$ \\
\hline $\begin{array}{l}\text { Объем резекции / энуклеации ПЖ, } \\
\text { см }\end{array}$ & $43,2 \pm 2,83$ & $40,6 \pm 2,80$ & 0,516 & $73,1 \pm 3,75$ & $75,2 \pm 3,68$ & 0,691 \\
\hline Срок п/о катетеризации МП, дни & $3,4 \pm 0,79$ & $6,2 \pm 1,09$ & $0,043^{*}$ & $5,3 \pm 1,01$ & $11,0 \pm 1,41$ & $0,002^{*}$ \\
\hline Срок госпитализации, дни & $5,0 \pm 0,95$ & $8,3 \pm 1,26$ & $0,049^{*}$ & $6,1 \pm 1,08$ & $12,5 \pm 1,50$ & $0,001^{*}$ \\
\hline $\begin{array}{l}\text { Объем кровопотери в ходе } \\
\text { операции, мл }\end{array}$ & $110 \pm 4,52$ & $190 \pm 6,05$ & $<0,001^{*}$ & $150 \pm 5,37$ & $340 \pm 7,81$ & $<0,001^{*}$ \\
\hline
\end{tabular}

* - различия достоверны, так как $\mathrm{p} \leq 0,05$.

подбирались ретроспективным методом по периоперационным данным архива историй болезни и протоколов операций с учетом статистической сопоставимости с основными группами пациентов, включенных в исследование, таблица 1. В контроль для сравнительного анализа с БТУР были включены данные 27 пациентов со средним

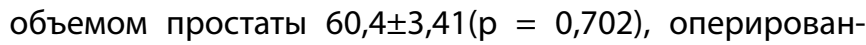
ные методом МТУР. Результаты ТУЭБ сравнивали с ОАЭ, проведенной 31 пациенту со средним объемом железы $91,1 \pm 4,05$ ( $p=0,851)$. Для количественной оценки достоверности межгрупповых различий средних и относительных показателей использовали непараметрический критерий Манна-Уитни, оценка качественных признаков проводилась с применением критерия Пирсона $\left(X^{2}\right)$, при уровне значимости различий $p \leq 0,05$.

Как видно из представленной таблицы, сравниваемые группы были статистически однородны ( $>>0,05)$, как по возрасту, частоте осложненных форм ДГП и сопутствующих заболеваний, так и по медианным значениям предоперационных урологических показателей: уровень простатоспецифического антигена (ПСА) в сыворотке крови, объем остаточной мочи по данным трансректального УЗИ, максимальная скорость мочевого потока по данным урофлоуметрии (PVR), балл по шкале оценки выраженности симптомов нижних мочевых путей — IPSS, балл по шкале оценки качества жизни в связи с расстройством мочеиспускания - QOL. Эти же урологические показатели, а также частота осложнений и повторных вмешательств оценивались в послеоперационном периоде по данным контрольных обследований пациентов через 1 и 6 месяцев после операций в целях изучения клинической эффективности новых хирургических методов.

\section{Результаты и обсужмение}

Средний объем удаленной ткани простаты был статистически равным для сравниваемых групп БТУР и МТУР $(p=0,516)$ и ТУЭБ и ОАЭ ( $p=0,691)$, что свидетельствует о равных возможностях сравниваемых хирургических методов в аспекте радикальности проведенных вмешательств, таблица 2. Среднее время выполнения БТУР и МТУР также различалось незначительно ( $p=0,605)$, время ТУЭБ было большим, чем ОАЭ в среднем в 1,5 раза ( $p<0,001)$, однако это не ухудшило остальные общие операционные результаты.

Как показано в таблице 2 биполярные методы отличаются существенно меньшей интраоперационной кровопотерей - БТУР в среднем в 1,7 раза, варьируя в промежутке от 50 до 150 мл, ТУЭБ - в 2,3 раза — от 50 до 170 мл, тогда как максимальная кровопотеря при МТУР достигала 250 мл ( $<<0,001)$, ОАЭ-450 мл $(p<0,001)$. В числе общих операционных показателей БТУР и ТУЭБ также обращает внимание достоверное снижение в сравнении с традиционными методами сроков послеоперационной катетеризации мочевого пузыря (МП) в 1,8-2,1 раза соответственно ( $p=0,043 ; p=0,002)$. Более быстрое восстановление самостоятельного мочеиспускания мы связываем с существенно меньшей инвазивностью новых методов и, как следствие, с минимальным количеством ранних послеоперационных геморрагических и инфекционно-воспалительных осложнений.

Раннее восстановление мочеиспускания при незначительном числе осложнений позволило значимо снизить сроки пребывания пациентов в стационаре, составившие в среднем 5,0 дней для БТУР (МТУР - 8,3 дня, $\mathrm{p}=$ $0,049)$ и 6,1 дней для ТУЭБ (ОАЭ - 12,5 дней, $\mathrm{p}=0,001$ ). Данный критерий следует считать особо важным достижением, поскольку уменьшение послеоперационного койко-дня позволяет экономить средства стационара.

В числе интраоперационных осложнений можно отметить 1 случай электроожоговой хирургической травмы в группе МТУР, обусловленный техническими недостатками монополярного электрода, таблица 3. В группе БТУР осложнений в ходе операций не возникло, что свидетельствует о безопасности и лучшей переносимости данного метода. Сравнение групп ТУЭБ и ОАЭ показало значимо меньшую инвазивность ново- 
Таблица 3. Осложнения оперативного лечения (\% в группе - P $\pm \mathrm{m}$ )

\begin{tabular}{|c|c|c|c|c|c|c|}
\hline $\begin{array}{l}\text { Метод } \\
\text { операции }\end{array}$ & $\begin{array}{l}\text { БТУР } \\
\mathrm{N}=29\end{array}$ & $\begin{array}{l}\text { MTУP } \\
\mathrm{N}=27\end{array}$ & $\mathbf{P}$ & $\begin{array}{l}\text { ТУЭБ } \\
N=27\end{array}$ & $\begin{array}{l}\text { OAЭ } \\
N=31\end{array}$ & $\mathbf{P}$ \\
\hline \multicolumn{7}{|c|}{ Интраоперационные осложнения } \\
\hline Всего, В том числе: & $0,0 \pm 0,00$ & $3,7 \pm 3,70$ & 0,322 & $11,1 \pm 6,16$ & $22,6 \pm 7,51$ & 0,243 \\
\hline массивное кровотечение & $0,0 \pm 0,00$ & $0,0 \pm 0,00$ & 1,000 & $3,7 \pm 3,70$ & $9,7 \pm 5,31$ & 0,362 \\
\hline гемотрансфузия & $0,0 \pm 0,00$ & $0,0 \pm 0,00$ & 1,000 & $3,7 \pm 3,70$ & $9,7 \pm 5,31$ & 0,362 \\
\hline $\begin{array}{l}\text { хирургическая травма, } \\
\text { электроожоги }\end{array}$ & $0,0 \pm 0,00$ & $3,7 \pm 3,70$ & 0,322 & $3,7 \pm 3,70$ & $3,2 \pm 3,17$ & 0,921 \\
\hline \multicolumn{7}{|c|}{ Ранние послеоперационные осложнения } \\
\hline Всего, в том числе: & $3,5 \pm 3,45$ & $14,8 \pm 6,97$ & 0,150 & $3,7 \pm 3,70$ & $25,8 \pm 7,86$ & 0,014 \\
\hline кровотечение & $3,5 \pm 3,45$ & $7,4 \pm 5,14$ & 0,525 & $0,0 \pm 0,00$ & $9,7 \pm 5,31$ & $0,047^{*}$ \\
\hline инфекционно-воспалительные & $3,5 \pm 3,45$ & $14,8 \pm 6,97$ & 0,150 & $3,7 \pm 3,70$ & $25,8 \pm 7,86$ & $0,014^{*}$ \\
\hline задержка мочи & $0,0 \pm 0,00$ & $7,4 \pm 5,14$ & 0,156 & $3,7 \pm 3,70$ & $16,1 \pm 6,61$ & 0,107 \\
\hline ТУР-синдром & $0,0 \pm 0,00$ & $3,7 \pm 3,70$ & 0,322 & $0,0 \pm 0,00$ & $0,0 \pm 0,00$ & 1,000 \\
\hline \multicolumn{7}{|c|}{ Поздние послеоперационные осложнения } \\
\hline Всего, в том числе: & $3,5 \pm 3,45$ & $14,8 \pm 6,97$ & 0,150 & $11,1 \pm 6,16$ & $9,7 \pm 5,31$ & 0,858 \\
\hline рубцовая деформация шейки МП & $0,0 \pm 0,00$ & $3,7 \pm 3,70$ & 0,322 & $3,7 \pm 3,70$ & $3,2 \pm 3,17$ & 0,921 \\
\hline стриктура уретры & $0,0 \pm 0,00$ & $7,4 \pm 5,14$ & 0,156 & $7,4 \pm 5,14$ & $6,5 \pm 4,41$ & 0,889 \\
\hline недержание мочи & $3,5 \pm 3,45$ & $3,7 \pm 3,70$ & 0,960 & $0,0 \pm 0,00$ & $3,2 \pm 3,17$ & 0,312 \\
\hline
\end{tabular}

* - различия достоверны, так как $\mathrm{p} \leq 0,05$.

Таблица 4. Послеоперационные показатели по данным урологического обследования в контрольные сроки (медиана в соответствующих единицах измерения)

\begin{tabular}{|c|c|c|c|c|c|c|}
\hline $\begin{array}{l}\text { Метод } \\
\text { операции }\end{array}$ & $\begin{array}{l}\text { БТУР } \\
N=29\end{array}$ & $\begin{array}{l}\text { MTУP } \\
N=27\end{array}$ & $\mathbf{P}$ & $\begin{array}{l}\text { ТУЭБ } \\
N=27\end{array}$ & $\begin{array}{l}\text { OAЭ } \\
N=31\end{array}$ & $\mathbf{P}$ \\
\hline \multicolumn{7}{|c|}{ Контрольное наблюдение через 1 мес. после операции } \\
\hline УзИ объем ПЖ, см³ & $20,6 \pm 1,96$ & $20,5 \pm 1,99$ & 0,972 & $28,5 \pm 2,34$ & $29,5 \pm 2,30$ & 0,762 \\
\hline Объем остаточной мочи, PVR, мл & $14,1 \pm 1,62$ & $16,0 \pm 1,75$ & 0,430 & $15,2 \pm 1,71$ & $22,4 \pm 2,01$ & $0,008^{*}$ \\
\hline Скорость потока мочи, Qmax, мл/сек & $16,3 \pm 1,74$ & $15,1 \pm 1,70$ & 0,624 & $14,3 \pm 1,66$ & $11,6 \pm 1,44$ & 0,225 \\
\hline Балл по шкале IPSS & $6,4 \pm 1,09$ & $7,9 \pm 1,23$ & 0,366 & $7,5 \pm 1,20$ & $12,8 \pm 1,52$ & $0,008^{*}$ \\
\hline Балл по шкале QOL & $2,2 \pm 0,64$ & $2,7 \pm 0,72$ & 0,606 & $2,0 \pm 0,62$ & $3,2 \pm 0,76$ & 0,226 \\
\hline \multicolumn{7}{|c|}{ Контрольное наблюдение через 6 мес. после операции } \\
\hline УЗИ объем ПЖ, см³ & $18,6 \pm 1,86$ & $17,9 \pm 1,86$ & 0,791 & $20,5 \pm 1,99$ & $21,2 \pm 1,95$ & 0,802 \\
\hline Объем остаточной мочи, PVR, мл & $5,0 \pm 0,96$ & $5,5 \pm 1,03$ & 0,724 & $5,2 \pm 1,00$ & $5,4 \pm 0,98$ & 0,887 \\
\hline Скорость потока мочи, Qmax, мл/сек & $19,1 \pm 1,88$ & $18,7 \pm 1,90$ & 0,882 & $18,8 \pm 1,90$ & $18,6 \pm 1,83$ & 0,940 \\
\hline Балл по шкале IPSS & $3,4 \pm 0,79$ & $3,8 \pm 0,86$ & 0,733 & $3,5 \pm 0,82$ & $4,1 \pm 0,86$ & 0,615 \\
\hline Балл по шкале QOL & $1,2 \pm 0,47$ & $1,4 \pm 0,52$ & 0,777 & $1,5 \pm 0,54$ & $1,7 \pm 0,55$ & 0,796 \\
\hline
\end{tabular}

* - различия достоверны, так как $\mathrm{p} \leq 0,05$.

го метода, поскольку массивных кровотечений, требующих гемотрансфузии, в данной группе было в 3 раза меньше. Однако подтвердить статистическое различие по показателю частоты интраоперационных осложнений не удалось вследствие малого количества наблюдений.

По количеству осложнений раннего послеоперационного периода следует отметить 5-7 кратное снижение их частоты в группах биполярнных трансуретральных вмешательств относительно традиционных хирурги- ческих методов. Кровотечение было единичным (3,5\%) в группе БТУР и не отмечалось в группе ТУЭБ, тогда как в группе МТУР их было 2 (7,4\%), и ОАЭ - 3 (9,7\%), $\mathrm{p}=0,047$. Инфекционно-воспалительные осложнения в группах БТУР и ТУЭБ были единичны, в группах сравнения значительно больше - 4 случая $(14,8 \%)$ в группе МТУР и $8(25,8 \%)$ в группе ОАЭ, $p=0,014$. По частоте задержки мочи после извлечения уретрального катетера также преимущество у биполярных методов. Кроме того, в группе МТУР наблюдался 1 случай (3,7\%) ТУР-синдрома (синдрома водной интоксикации), характерный для дан- 
ного хирургического метода вследствие использования монополярного электрода и гипертонического раствора в качестве ирриганта. Применение биполярной техники ТУР с применением для ирригации физиологического раствора исключает возможность развития данного грозного осложнения.

Отсроченных послеоперационных осложнений обструктивного характера в группе БТУР не отмечено, МТУР - 2 случая $(7,4 \%)$ рубцовой деформации шейки мочевого пузыря и 1 (3,7\%) - стриктуры уретры, но без статистически доказанной разности. Случаев недержания мочи было по одному в каждой группе. В группах ТУЭБ и ОАЭ значимой разности по частоте поздних осложнений значимых различий не установлено. Повторных хирургических вмешательств, связанных с коррекцией недержания мочи и устранением рубцовых осложнений, было по 1 случаю в группах БТУР и МТУР, в остальных исследуемых группах не наблюдалось.

Послеоперационная динамика урологических показателей и измерений по шкалам IPSS и QOL, отслеженная по результатам контрольных обследований пациентов через 1 и 6 мес. после выписки из стационара, независимо от метода операции свидетельствовала о высокой клинической эффективности проведенного хирургического лечения, таблица 4. Сопоставление с исходными данными пациентов показало 3-х кратное снижение объема простаты через месяц и 4-х кратное - через 6 месяцев после операции. Как продемонстрировано в таблице, объем остаточной мочи значимо снижается, а скорость мочеиспускания значимо возрастает в первый послеоперационный месяц, и эти показатели достигают возрастной нормы через полгода после операций. Аналогичная картина наблюдается и по показателям опросных шкал IPSS и QOL. B то же время следует отметить более быструю месячную динамику некоторых средних показателей (объем остаточной мочи и балл по шкале IPSS), обеспечившую их статистически значимую разность между группами пациентов, оперированных методами ТУЭБ и ОАЭ. Выявленное различие следует трактовать как более быстрое восстановление нарушенных параметров мочеиспускания и общего состояния пациентов после применения нового эндохирургического метода.

\section{ЛИТЕРАТУРА}

1. Гордеев В.В., Антонов А. Г., Писаренко М. Ю., и др. Биполярная трансуретральная резекция в физиологическом растворе при осложненных формах доброкачественной гиперплазии предстательной железы // Тихоокеанский медицинский журнал. 2016. № 1. С. 79-81.

2. Камалов А.А., Севрюков Ф. А. Изучение эффективности биполярной трансуретральной резекции аденомы простаты средних и малых размеров // Современные технологии в медицине. 2012. № 4. С. 68-72.

3. Севрюков Ф.А., Камаев И. А., Гриб М. Н., Перевезенцев Е. А. Основные аспекты обеспечения высококвалифицированной урологической медицинской помощью больных с доброкачественной гиперплазией предстательной железы // Российский медико-биологический вестник имени академика И. П. Павлова.—2011. -Том 19. -№ 1. -С. 109-113.

4. Севрюков Ф.А., Камаев И. А., Малинина 0. Ю., Елина Ю. А., Гриб М. Н., Перевезенцев Е. А. Возрастные особенности заболеваемости мужчин болезнями мочеполовой системы // Медицинский альманах. 2011. № 4 (17). С. $21-23$.

5. Севрюков Ф. А. Качество жизни больных после трансуретральной энуклеации аденомы предстательной железы больших размеров // Андрология и генитальная хирургия. 2012. Т. 13. № 3. С. 42-46.

6. Севрюков Ф.А., Пучкин А. Б., Крупин В. Н., Чебыкин А. В., Сорокин Д. А., Карпухин И. В., Малинина 0. Ю., Зорин Д. Г. Трансуретральная электрохирургия нового поколения (TURIS) в лечении заболеваний нижних мочевых путей и простаты // Урология. 2007. № 3. С. $28-35$.

7. Севрюков Ф.А., Серебряный С. А. Случай успешной трансуретральной энуклеации аденомы простаты очень больших размеров // Андрология и генитальная хирургия. 2012. Т. 13. № 3. С. 101-103.

8. Урология. Российские клинические рекомендации / под ред. Ю. Г. Аляева, П. В. Глыбочко, Д. Ю. Пушкаря. М.: ГЭОТАР Медиа, 2016. 496 с.

9. Chen S., Zhu L., Cai J., et al. Plasmakinetic enucleation of the prostate compared with open prostatectomy for prostates larger than 100 grams: A randomized noninferiority controlled trial with long-term results at 6 years. // Eur. Urol. 2014. № 66. P. 284-291.

10. EAU Guidelines on the Assessment of Non-Neurogenic Male Lower Urinary Tract Symptoms Including Benign Prostatic Obstruction / Ed. S. A. Kaplan [et al.] // J. Urol. 2016. № 196(6). P. 1712-4.

11. Geavlete B., Bulai C., Ene C., at al. Bipolar vaporization, resection, and enucleation versus open prostatectomy: optimal treatment alternatives in large prostate cases? // J. Endourol. 2015. № 29(3). P. 323-31.

12. Gilling P. TURP remains a safe and effective alternative for benign prostatic hyperplasia (BPH) surgery // BJU Int., 2014. № 113(1). P. 5-6.

13. Li M., Qiu J., Hou Q. et al. Endoscopic enucleation versus open prostatectomy for treating large benign prostatic hyperplasia: a meta-analysis of randomized controlled trials. PLoS One 2015. № 10(3): e0121265.

14. Ou R., Deng X., Yang W., et al. Transurethral enucleation and resection of the prostate vs transvesical prostatectomy for prostate volumes $>80 \mathrm{~mL}$ : a prospective randomized study // BJU Int. 2013. № 112. P. 239-245.

15. Patel N. D., Parsons J. K. Epidemiology and etiology of benign prostatic hyperplasia and bladder outlet obstruction // Indian J. Urol. 2014. № 30(2). P. 170-176. 
16. Qiu J., Hou Q., et al. Endoscopic enucleation versus open prostatectomy for treating large benign prostatic hyperplasia: a meta-analysis of randomized controlled trials // PLOS One 2015. № 10(3): e0121265.

17. Rao J. M., Xiao H. J., Ren Y.X., et al. Did prostate size affect the complication and outcome of plasmakinetic enucleation of the prostate? // International Urology and Nephrology. 2014. № 46(11). P. 2063-70.

18. Stucki P., Marini L., Mattei A., at al. Bipolar versus monopolar transurethral resection of the prostate: a prospective randomized trial focusing on bleeding complications // J. Urol., 2015. № 193(4). P. 1371-76.

19. Tang Y., Li J., Pu C., at al. Bipolar transurethral resection versus monopolar transurethral resection for benign prostatic hypertrophy: a systematic review and metaanalysis // J. Endourol. 2014. № 28(9). P.1107-14.

20. Yu X., Elliott S. P., Wilt T. J., McBean A. M. Practice patterns in benign prostatic hyperplasia surgical therapy: the dramatic increase in minimally invasive technologies // J. Urol. 2008. № 180(1). P. 241-5.

○ Кононов Павел Алексеевич ( pavkonon@gmail.com ), Курнаков Антон Михайлович.

Журнал «Современная наука: актуальные проблемы теории и практики»

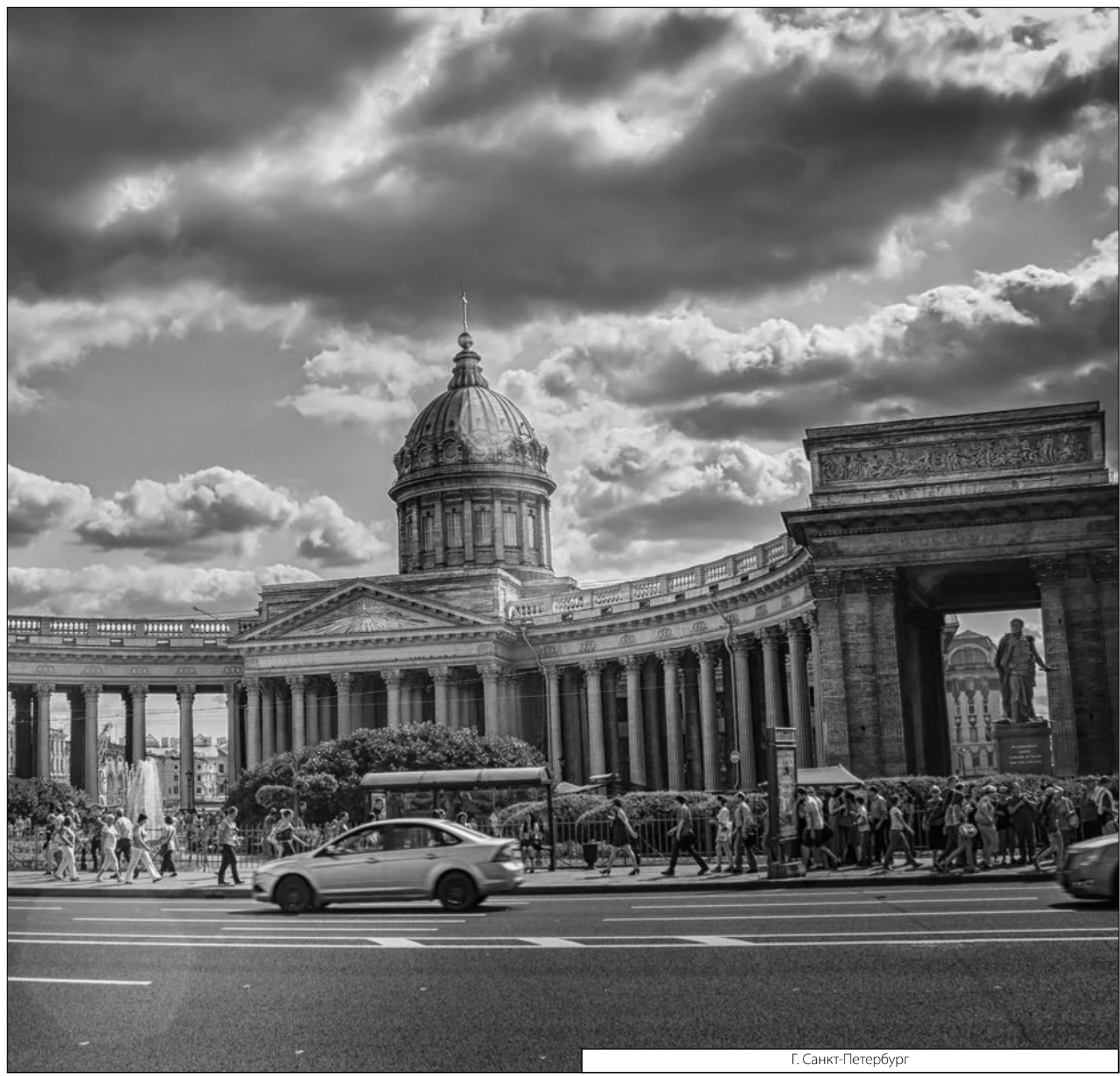

